\title{
Equilibrium shape of an anisotropic crystal confined between two planar parallel walls
}

\author{
Jean-Christophe Géminard and Patrick Oswald \\ Ecole Normale Supérieure de Lyon, 69364 Lyon Cedex 07, France
}

(Received 15 October 1996)

\begin{abstract}
Experimentally, the surface-free-energy anisotropy of a crystal-liquid interface is usually inferred from the surface-shape anisotropy of a solid germ at equilibrium with the melt. However, the crystal is usually not free. For example, in thin samples, the crystal touches the two limiting glass plates: Here, we analyze theoretically how the shape anisotropy of the crystal depends on the wetting conditions in this particular geometry. We calculate the equilibrium shape of an anisotropic crystal (of uniaxial, cubic, or hexagonal symmetry) confined between two planar parallel walls as a function of its size and of the wetting conditions on the walls. We find an analytic solution for the shape of the meniscus in the sample thickness and its anisotropy in the midplane parallel to the walls in the limit of very large radius and vanishing surface tension anisotropy. In other cases, a numerical solution is given. Neglecting the meniscus effect leads to errors as large as $25 \%$ for the inferred anisotropy. [S1063-651X(97)08904-6]
\end{abstract}

PACS number(s): 68.10.Cr, 68.35.Bs

\section{INTRODUCTION}

The shape of an isolated crystal in equilibrium with its melt or its vapor (and, more generally of a germ of phase 1 in equilibrium with phase 2) is deeply related to its surface properties and is usually obtained by minimizing its total surface free energy. Such a minimization leads to the famous geometrical Wulff construction that allows us to construct the equilibrium shape of the crystal from its threedimensional (3D) polar plot $\gamma(\hat{n})$. Here $\gamma$ is the surface energy and $\hat{n}$ is the unit vector normal to the interface [1-5]. On the other hand, this construction does not apply when the crystal is in contact with a wall, which is usually the case in experiments. In practice, the crystal lies on a substrate or is sandwiched between two parallel glass plates. In studies of the shape of liquid crystal domains, the latter geometry is often used because the glass can be treated in order to orient the molecules. Having well-defined boundary conditions on the glass plates is crucial for obtaining a monodomain (i.e., a domain in which the director field has everywhere the same orientation). Also, the presence of the plates prevents germ distorsion effects such as boojums in nematic liquid crystals [6,7], focal conics in smectic liquid crystals [8-11] (the famous "bâtonnets de Friedel" [9]), or developable domains in columnar mesophases [12-14]. In these latter cases, the problem is much more difficult to treat because elasticity must be taken into account in the calculations [15]. One consequence is that the Wulff construction cannot be applied in its usual form $[16,17]$.

In the following, we assume that the material (a crystal, liquid crystal, or simple liquid) is sandwiched between two planar parallel walls that impose a well-defined orientation. For this reason, we completely neglect elastic effects, but we take into account the wetting conditions on the two limiting surfaces. Our purpose is to find the equilibrium shape of the germ in these conditions. We again point out that, in the confined 3D case, the Wulff construction cannot be used and that the equilibrium shapes are no longer self-similar.

Solving this problem theoretically is important for practical reasons because the surface-free-energy anisotropy of many materials (such as plastic crystals or liquid crystals) is often deduced from measurements of the equilibrium shape of germs sandwiched between two glass plates. It is usually assumed that the shape anisotropy is equal to that of the surface free energy. This is only true when the (2D) Wulff construction applies (i.e., when the angle between the interface and the limiting walls is equal to $\pi / 2$ ) [18]. Otherwise, this result does not hold and there is no reason to assume that both anisotropies are equal. Our goal, here, is to calculate for materials of classical crystalline symmetries, how the shape anisotropy of a germ depends on its size, on the surface tension anisotropy, and on the contact angle of the interface with the limiting walls. We shall show that in typical experimental situations neglecting the effects of anisotropy upon the meniscus can lead to errors of the order of $10 \%$.

We also emphasize that solving this problem is important in the study of crystal growth. Indeed, it is known that the marginal stability constant $\sigma^{*}$ of a stationary growing dendrite is a universal function of the surface energy anisotropy $\epsilon$ [19-22]. Thus, an accurate measurement of $\epsilon$ is of a great importance to compare the experimental results with the theoretical predictions. This is particularly important since there often exists a discrepancy between experimental and theoretical values of $\sigma^{*}$ (calculated by using the experimental value of $\epsilon)[23,24]$.

The organization of this paper is as follows: In Sec. II, we formulate the general problem of finding the equilibrium shape of an anisotropic germ in contact with two parallel planar surfaces. We calculate, in Sec. III, an analytical expression of the shape anisotropy of the germ in the limit of vanishing surface energy anisotropy $\epsilon \rightarrow 0$ and $\rho_{0} \rightarrow \infty$ where $\rho_{0}$ is the mean radius of the germ in the midplane parallel to the walls. Next, we compute, in Sec. IV, the shape anisotropy of a germ of finite radius $\rho_{0}$ but of vanishing anisotropy. Finally, we give, in Sec. V, some numerical results obtained for germs of both finite radius and finite surface energy anisotropy.

\section{GENERAL FORMULATION OF THE PROBLEM}

We consider the case of an anisotropic material (a crystal or a liquid crystal), in equilibrium with a liquid (its melt for 


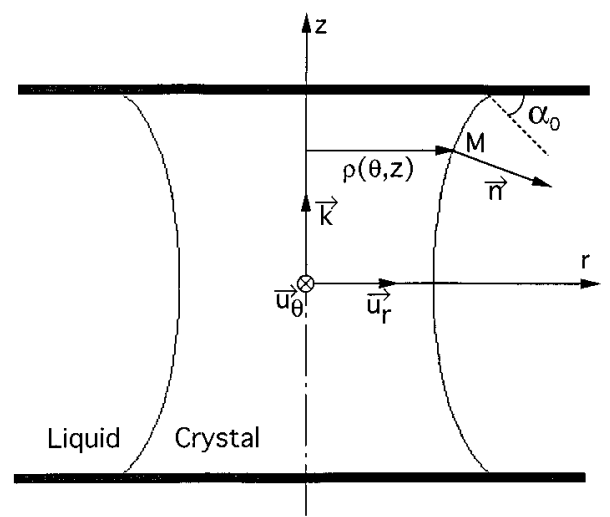

FIG. 1. Schematic representation of the meniscus.

example), confined between two parallel planes separated by a distance $d$ (Fig. 1). We describe the interface in cylindrical coordinates $\left(\vec{u}_{r}, \vec{u}_{\theta}, \vec{k}\right)$. Because the sample thickness $d$ of the sample is the only length scale, all lengths scale by $d$. Thus, the boundaries are at $z= \pm \frac{1}{2}$. We define the interface points via two variables $\theta$ and $z$ :

$$
\overrightarrow{O M}=\rho(\theta, z) \vec{u}_{r}+z \vec{k}
$$

The interface is characterized by its anisotropic surface free energy $\gamma(\hat{n})$, where $\hat{n}$ is the unit vector normal to the interface:

$$
\hat{n}=\frac{\rho \vec{u}_{r}-\rho_{\theta} \vec{u}_{\theta}-\rho \rho_{z} \vec{k}}{\sqrt{\rho^{2}\left(1+\rho_{z}^{2}\right)+\rho_{\theta}^{2}}} .
$$

We assume, for simplicity, that the wetting conditions are the same on both surfaces and that one of the crystallographic axis is perpendicular to the limiting walls. Let $\gamma_{c s}\left(\gamma_{l s}\right)$ be the surface free energies of the crystal (liquid) with the substrate. In the following, we assume that both quantities are constant. The total surface free energy, calculated inside a cylinder of arbitrary radius $r_{0}$ surrounding the meniscus, is given by

$$
\begin{aligned}
E_{s}= & \int_{0}^{2 \pi} \int_{-1 / 2}^{1 / 2} \gamma d S+\int_{0}^{2 \pi} \int_{0}^{\rho(\theta, 1 / 2)} \gamma_{c s} r d \theta d r \\
& +\int_{0}^{2 \pi} \int_{\rho(\theta, 1 / 2)}^{r_{0}} \gamma_{l s} r d \theta d r+\int_{0}^{2 \pi} \int_{0}^{\rho(\theta,-1 / 2)} \gamma_{c s} r d \theta d r \\
& +\int_{0}^{2 \pi} \int_{\rho(\theta,-1 / 2)}^{r_{0}} \gamma_{l s} r d \theta d r
\end{aligned}
$$

where $d S=\sqrt{\rho^{2}\left(1+\rho_{z}^{2}\right)+\rho_{\theta}^{2}} d \theta d z$. The calculation of the last four integrals gives

$$
\begin{aligned}
E_{s}= & \int_{0}^{2 \pi} \int_{-1 / 2}^{1 / 2} \gamma d S+\Delta \gamma \int_{0}^{2 \pi} \frac{\rho^{2}\left(\theta, \frac{1}{2}\right)+\rho^{2}\left(\theta,-\frac{1}{2}\right)}{2} d \theta \\
& +2 \pi \gamma_{l s} r_{0}^{2},
\end{aligned}
$$

where $\Delta \gamma=\gamma_{c s}-\gamma_{l s}$. Below, we consider only the excess of surface free energy and thus neglect the last term in Eq. (4).
We also scale all the surface free energies by $\gamma_{0}$, the mean value of $\gamma(\hat{n})$ over all the possible orientations and we define

$$
\phi=\gamma \sqrt{\rho^{2}\left(1+\rho_{z}^{2}\right)+\rho_{\theta}^{2}} .
$$

By definition, the volume $V$ of the crystal is given by

$$
V=\int_{0}^{2 \pi} \int_{-1 / 2}^{1 / 2} \frac{\rho^{2}(\theta, z)}{2} d \theta d z .
$$

The equilibrium shape of the crystal minimizes the surface free energy $E_{s}$. If the volume $V$ is constant, the functional to minimize is $E_{s}-\lambda V$, where $\lambda$ is a Lagrange multiplier. This gives

$$
\delta E_{s}-\lambda \delta V=0 .
$$

A straightforward calculation shows that $\rho(\theta, z)$ obeys the volume equation

$$
\frac{\partial \phi}{\partial \rho}-\frac{d}{d \theta} \frac{\partial \phi}{\partial \rho_{\theta}}-\frac{d}{d z} \frac{\partial \phi}{\partial \rho_{z}}-\lambda \rho=0
$$

with the two boundary conditions

$$
\frac{\partial \phi}{\partial \rho_{z}}\left(\theta, \pm \frac{1}{2}\right)+\Delta \gamma \rho\left(\theta, \pm \frac{1}{2}\right)=0 .
$$

These boundary conditions give the contact angle $\alpha_{0}$ [i.e., the angle between the normal $\hat{n}$ to the interface and the limiting walls (Fig. 1)].

The problem now consists in solving this set of equations for a given function $\gamma(\hat{n})$. Equation (8) expresses, in cylindrical coordinates, the local mechanical equilibrium of the interface and can be rewritten in the usual form $[2,4]$ :

$$
\left(\frac{\gamma+\gamma^{\prime \prime}}{\mathfrak{R}_{1}}\right)_{\Pi_{1}}+\left(\frac{\gamma+\gamma^{\prime \prime}}{\mathfrak{R}_{2}}\right)_{\Pi_{2}}=-\lambda,
$$

where $\left(\Pi_{1}\right)$ and $\left(\Pi_{2}\right)$ are the two principal planes of curvature and, $\mathfrak{R}_{1}$ and $\mathfrak{R}_{2}$, the two principal radii of curvature. Each second derivative of the surface free energy is calculated with respect to a polar angle taken in the considered plane of curvature. The boundary conditions (9), known as the Young relation $[3,25]$, relate the contact angle to the surface free energies on the limiting walls.

Let us now determine the surface-shape anisotropy in the limiting case of vanishing surface-free-energy anisotropy and large radius.

\section{ASYMPTOTIC BEHAVIOR AT LARGE RADIUS $\rho_{0} \rightarrow \infty$ AND SMALL ANISOTROPY $\epsilon \rightarrow 0$}

We assume that the mean radius $\rho_{0}$ of the meniscus in the plane $z=0$ is much larger than the sample thickness $\left(\rho_{0} \gg 1\right)$. We assume the following form for $\rho(\theta, z)$ (Fig. 2):

$$
\rho(\theta, z)=r_{p}(\theta)+r_{t}(\theta, \nu) \cos \nu-r_{t}(\theta, 0) .
$$

The polar angle $\nu$ satisfies in each plane $\theta=$ const the relation

$$
z=r_{t}(\theta, \nu) \sin \nu .
$$




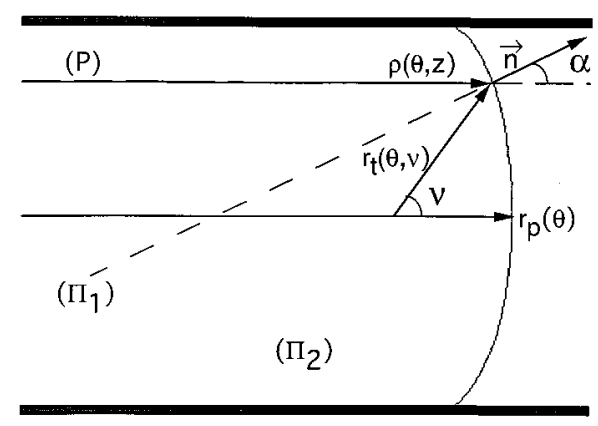

FIG. 2. Schematic representation of the meniscus in a plane of symmetry $\theta=k \pi / n . \Pi_{1}$ and $\Pi_{2}$ are two principal planes of curvature.

With this choice, the horizontal and the vertical scales are decoupled because $r_{p}$ scales like $\rho_{0}$ whereas $r_{t}$ scales like the thickness (for $\alpha_{0} \neq 0$ ). The position $\rho(\theta, 0)=r_{p}(\theta)$ $-r_{t}(\theta, 0)$ of the origin of the vector $\vec{r}_{t}$ is a priori not known and can be later deduced from the solution we determine below. We point out that experimentally (Fig. 3) the contour of the germ the most contrasted in the microscope corresponds to the vertical part of the meniscus described by the function $r_{p}(\theta)$.

In Sec. III A, we determine the shape $r_{t}(\theta, \nu)$ of the meniscus in a vertical plane $(\theta=$ const $)$ chosen as a plane of symmetry of the crystal, and we show that the wetting conditions on the limiting walls impose a relation between the Lagrange parameter $\lambda$ and the radius of curvature of the meniscus in the horizontal plane. We then use this result to determine $r_{p}(\theta)$ in the limit of vanishing surface-free-energy anisotropy.

\section{A. Shape of the meniscus in a vertical plane}

From now on, we assume that the crystal has an $n$-fold axis perpendicular to the limiting walls. Here, we choose as

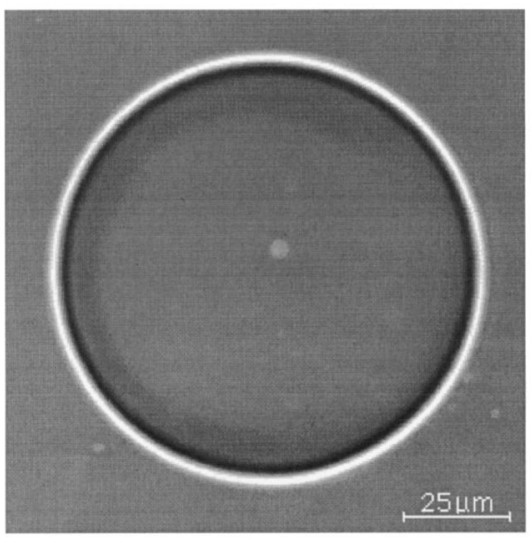

FIG. 3. Experimental observation of a columnar hexagonal mesophase through the microscope. The "Becke", line [30] surrounding the germ follows the vertical part of the meniscus. The material is the discotic liquid crystal $\mathrm{C}_{8} \mathrm{HET}$ (hexa-ester of triphenylene) [28]. It is sandwiched between two parallel glass plates that anchor the molecular columns (i.e., the $C_{6}$ axis) perpendicular to them. The sample thickness $d$ is close to $3 \mu \mathrm{m}$ and the radius $\rho_{0}$ equals 30 $\mu \mathrm{m}$. The contact angle $\alpha_{0}$ is about $60^{\circ}$. The shape anisotropy $\epsilon_{6}^{\prime}$ measured on such a photograph is small, of the order of $3 \times 10^{-3}$. a plane $\left(\Pi_{2}\right)$ a plane of symmetry of the crystal $\theta=k \pi / n$ ( $k$ integer). For a given value of $\nu$, the corresponding plane $\left(\Pi_{1}\right)$ is the plane perpendicular to $\left(\Pi_{2}\right)$ that contains the normal $\hat{n}$ to the interface. The two surface stiffnesses are then

$$
\left(\gamma+\gamma^{\prime \prime}\right)_{\Pi_{1}}=\left(\gamma+\frac{\gamma_{\beta \beta}}{\cos ^{2} \alpha}-\tan \alpha \gamma_{\alpha}\right)_{\beta=k \pi / n}
$$

and

$$
\left(\gamma+\gamma^{\prime \prime}\right)_{\Pi_{2}}=\left(\gamma+\gamma_{\alpha \alpha}\right)_{\beta=k \pi / n},
$$

where $\alpha$ is the angle between the normal $\hat{n}$ and the horizontal plane $(P)$ and $\beta$ is the angle that $\hat{n}$ makes with the $x$ axis in the horizontal plane $(P)$ [the plane $\left(\Pi_{2}\right)$ being chosen as a plane of symmetry of the crystal $\beta=\theta]$. The radius of curvature $\Re_{1}$ can also be expressed as a function of the radius of curvature $\mathfrak{R}_{h}$ in the horizontal plane $(P)$ :

$$
\mathfrak{R}_{1}=\frac{\mathfrak{R}_{h}}{\cos \alpha} .
$$

If we remember that $\rho_{\theta}=0$ in a symmetry plane, we have

$$
\mathfrak{R}_{h}=\rho+\rho_{\theta \theta}=r_{p}(\theta)+r_{p}^{\prime \prime}(\theta) .
$$

The second equality is valid if $r_{t} / r_{p} \ll 1$ (which can be checked a posteriori). As for $\mathfrak{R}_{2}$, it is given by the usual relation:

$$
\mathfrak{R}_{2}=\left(\frac{r_{t}+r_{t}^{\prime \prime}}{\left(1+r_{t}^{\prime 2}\right)^{3 / 2}}\right)_{\theta=k \pi / n},
$$

where primes denote the derivatives with respect to $\nu$. We can now rewrite the local equilibrium equation (10) in a plane of symmetry $\left(\Pi_{2}\right)$ :

$$
\begin{aligned}
& \left(\frac{\gamma \cos ^{2} \alpha+\gamma_{\beta \beta}-\gamma_{\alpha} \sin \alpha \cos \alpha}{\mathfrak{R}_{h} \cos \alpha}\right)_{\beta=k \pi / n}+\left(\frac{\gamma+\gamma_{\alpha \alpha}}{\mathfrak{R}_{2}}\right)_{\beta=k \pi / n} \\
& \quad=-\lambda .
\end{aligned}
$$

For a given $\theta$ (or $\beta$ ), $r_{t}(\theta, \nu)$ satisfies this differential equation in which $\Re_{h}$ and $\lambda$ remain constant. To first order in $r_{t} / r_{p}$, this equation becomes

$$
\begin{aligned}
& \left(\frac{\left(\gamma+\gamma_{\alpha \alpha}\right)\left(1-\frac{\gamma \cos ^{2} \alpha+\gamma_{\beta \beta}-\gamma_{\alpha} \sin \alpha \cos \alpha}{\lambda \Re_{h} \cos \alpha}\right)}{\mathfrak{R}_{2}}\right)_{\beta=k \pi / n} \\
& \quad=-\lambda
\end{aligned}
$$

Let $\widetilde{\gamma}_{k}(\alpha)$ be a solution (even, for symmetry reasons) to the following equation: 


$$
\widetilde{\gamma}_{k}+\widetilde{\gamma}_{k}^{\prime \prime}=\left[\left(\gamma+\gamma_{\alpha \alpha}\right)\left(1-\frac{\gamma \cos ^{2} \alpha+\gamma_{\beta \beta}-\gamma_{\alpha} \sin \alpha \cos \alpha}{\lambda \Re_{h} \cos \alpha}\right)\right]_{\beta=k \pi / n} .
$$

We emphasize that $\widetilde{\gamma}_{k}(\alpha)$ is defined modulo a function of the form $A \cos \alpha$, which does not play any role. Then, the shape of the meniscus in a plane of symmetry $\Pi_{2}$ is given by solving the generic equation

$$
\frac{\widetilde{\gamma}_{k}+\vec{\gamma}_{k}^{\prime}}{\Re_{2}}=-\lambda
$$

Its solution is $[2,4,5]$

$$
\begin{gathered}
r_{t}(\nu)=-\frac{1}{\lambda} \sqrt{\widetilde{\gamma}_{k}^{2}(\alpha)+\widetilde{\gamma}_{k}^{2}(\alpha),} \\
\nu=\alpha+\arctan \frac{\widetilde{\gamma}^{\prime}(\alpha)}{\widetilde{\gamma}(\alpha)} .
\end{gathered}
$$

This solution must satisfy the geometrical condition (12) on the wall $z=\frac{1}{2}$ (idem on the opposite one) which gives, using Eqs. (21) and (22),

$$
\widetilde{\gamma}_{k}\left(\alpha_{0 k}\right) \sin \alpha_{0 k}+\widetilde{\gamma}_{k}^{\prime}\left(\alpha_{0 k}\right) \cos \alpha_{0 k}=-\frac{\lambda}{2},
$$

where $\alpha_{0 k}$ is the contact angle (depending on the $k$ plane chosen) given by the generalized Young relation [3,25]:

$$
\gamma\left(\alpha_{0 k}, k \frac{\pi}{n}\right) \sin \alpha_{0 k}+\gamma^{\prime}\left(\alpha_{0 k}, k \frac{\pi}{n}\right) \cos \alpha_{0 k}=\Delta \gamma .
$$

For a given value of the radius of curvature $\Re_{h}$ in the horizontal plane $(P)$, the shape $r_{t}(\nu)$ of the meniscus in the vertical plane $\left(\Pi_{2}\right)$ is determined by Eqs. (21) and (22), where $\lambda$ must satisfy the boundary condition [Eq. (23)]. Thus, in the limit of large $\rho_{0}$, we have determined a relation between $\lambda$ and $\Re_{h}$. We emphasize that, up to this point, we have made no assumption as to the exact form of the surface free energy $\gamma$.

\section{B. Shape $r_{p}(\theta)$ of the meniscus in the horizontal plane in the limit of vanishing anisotropy}

To continue the calculation analytically, we now need to assume that the shape of the germ can be written in the form (first term of the Fourier expansion)

$$
r_{p}(\theta)=\rho_{0}\left(1+\epsilon_{n}^{\prime} \cos n \theta\right) \quad \text { with } \epsilon_{n}^{\prime} \ll 1 .
$$

This will be the case if the surface free energy can similarly be rewritten in the form

$$
\gamma(\beta, \alpha)=F(\alpha)+\epsilon_{n} G(\alpha) \cos n \beta,
$$

with $F(0)=G(0)=1$ and $\epsilon_{n} \ll 1$. Note that we do not make any further assumptions about the exact form of $F(\alpha)$ and
$G(\alpha)$. We now sketch the analytical calculation of the shape anisotropy of the germ $\epsilon_{n}^{\prime}$ as a function of $\epsilon_{n}$ and $\alpha_{0}$ in the limit $\epsilon_{n} \rightarrow 0$.

(i) First, we calculate for each value of $k$ (in practice for $k=0$ and $k=1)$ the value of the contact angle $\alpha_{0 k}$ by Eq. (24).

(ii) Second, we integrate the second-order differential equation [Eq. (19)] for each value of $k$. Recall that by definition of $r_{p}(\theta)$, we have

$$
\mathfrak{R}_{h}=\rho_{0}\left[1+\epsilon_{n}^{\prime}(-1)^{k}\left(1-n^{2}\right)\right] .
$$

(iii) Third, we substitute for $\alpha_{0 k}$ and $\widetilde{\gamma}_{k}$ from the previous calculations into the geometrical relation [Eq. (23)]. This gives two equations of the form (to first order in $1 / \rho_{0}$ )

$$
-\lambda=2 \Delta \gamma+\frac{1}{\rho_{0}}\left[f\left(\epsilon_{n}^{\prime}, \epsilon_{n}, \alpha_{0}\right) \pm g\left(\epsilon_{n}^{\prime}, \epsilon_{n}, \alpha_{0}\right)\right]
$$

We define $\alpha_{0}$ as the contact angle calculated in the limit $\epsilon_{n}=0$. According to Eqs. (24) and (26), this angle is the solution of the equation

$$
F\left(\alpha_{0}\right) \sin \alpha_{0}+F^{\prime}\left(\alpha_{0}\right) \cos \alpha_{0}=\Delta \gamma \text {. }
$$

At equilibrium, $\lambda$ must be a constant so that [see Eq. (28)]
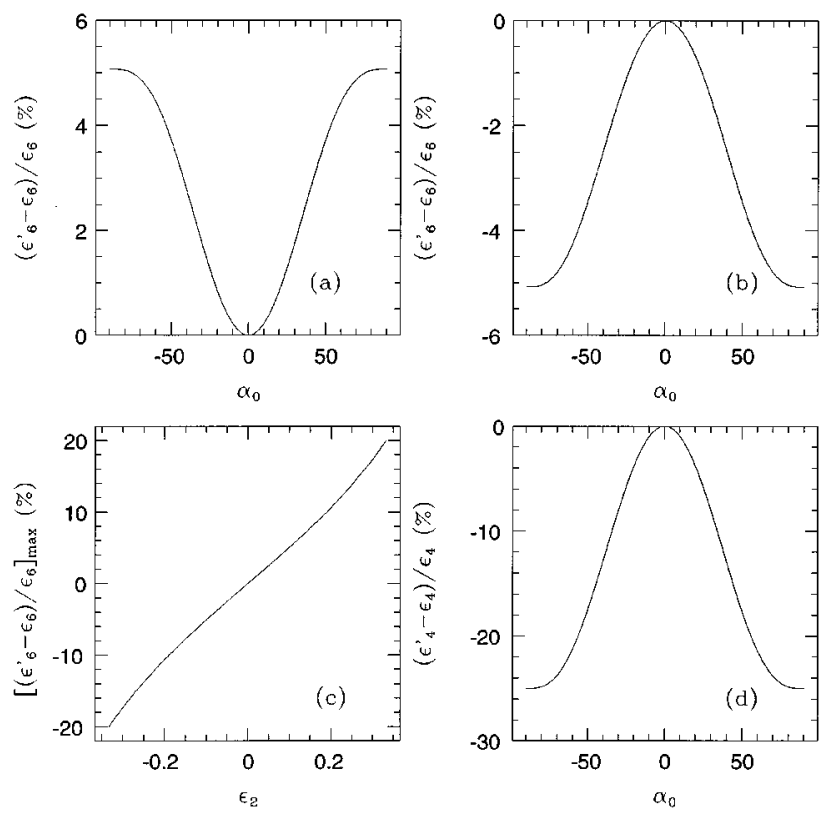

FIG. 4. Shape anisotropy at large radius $\rho_{0} \rightarrow \infty$ and vanishing anisotropy $\epsilon_{n} \rightarrow 0$. Relative anisotropy $\left(\epsilon_{6}^{\prime}-\epsilon_{6}\right) / \epsilon_{6}$ of a hexagonal crystal when its twofold anisotropy $\epsilon_{2}=0.1$ (a) and $\epsilon_{2}=-0.1$ (b). Maximal value of $\left(\epsilon_{6}^{\prime}-\epsilon_{6}\right) / \epsilon_{6}$ (reached when $\left.\alpha_{0}= \pm \pi / 2\right)$ as a function of $\epsilon_{2}(\mathrm{c})$. Relative anisotropy $\left(\epsilon_{4}^{\prime}-\epsilon_{4}\right) / \epsilon_{4}$ of a cubic crystal as a function of the contact angle $\alpha_{0}(\mathrm{~d})$. 


$$
g\left(\epsilon_{n}^{\prime}, \epsilon_{n}, \alpha_{0}\right)=0
$$

This equation formally gives the shape anisotropy $\epsilon_{n}^{\prime}$ as a function of $\epsilon_{n}$ and of the contact angle $\alpha_{0}$. Notice that $\lambda$ tends to $-2 \Delta \gamma$ (in units of $\gamma_{0} / d$ ) at infinite radius. Let us now consider a few examples for which we have done the complete calculation.

\section{Uniaxial crystal in planar orientation}

The simplest case corresponds to a uniaxial crystal (for example, a nematic liquid crystal in planar orientation). Setting the $x$ axis parallel to the $C_{\infty}$ axis, we have a surface free energy that is

$$
\gamma(\alpha, \beta)=\left(1-\epsilon_{2} \sin ^{2} \alpha\right)+\epsilon_{2}\left(\cos ^{2} \alpha\right) \cos 2 \beta .
$$

The calculation shows that, in this case, the shape anisotropy equals the surface energy anisotropy:

$$
\lim _{\substack{\rho_{0} \rightarrow \infty \\ \epsilon_{2} \rightarrow 0}} \frac{\epsilon_{2}^{\prime}-\epsilon_{2}}{\epsilon_{2}}=0
$$

\section{Cubic crystal}

Another interesting case is that of a cubic crystal with a $C_{4}$ axis perpendicular to the walls. Many plastic crystals, such as succinonitrile or pivalic acid (commonly used in growth experiments), have cubic symmetry. By taking the $x$ axis parallel to a $C_{4}$ axis, we have [24]

$$
\begin{aligned}
\gamma(\alpha, \beta)= & 1+\epsilon_{4}\left[4\left\{\cos ^{4} \alpha\left(\cos ^{4} \beta+\sin ^{4} \beta\right)+\sin ^{4} \alpha\right\}-3\right] \\
= & \left(1-3 \epsilon_{4}+3 \epsilon_{4} \cos ^{4} \alpha+4 \epsilon_{4} \sin ^{4} \alpha\right) \\
& +\epsilon_{4}\left(\cos ^{4} \alpha\right) \cos 4 \beta,
\end{aligned}
$$

and we calculate

$$
\lim _{\substack{\rho_{0} \rightarrow \infty \\ \epsilon_{4} \rightarrow 0}} \frac{\epsilon_{4}^{\prime}-\epsilon_{4}}{\epsilon_{4}}=-\frac{1}{8} \frac{4 \alpha_{0}-\sin 4 \alpha_{0}}{2 \alpha_{0}+\sin 2 \alpha_{0}} .
$$

The shape anisotropy is now different from the surface energy anisotropy and depends on the contact angle $\alpha_{0}$ (but not on its sign). We also see that the correction $\left(\epsilon_{4}^{\prime}-\epsilon_{4}\right) / \epsilon_{4}$ has a maximum equal to $25 \%$ for $\alpha_{0}= \pm \pi / 2$. This function is plotted in Fig. 4 versus $\alpha_{0}$.

\section{Hexagonal crystal}

Finally, we did the calculation for a hexagonal crystal whose $C_{6}$ axis is perpendicular to the walls. The $x$ axis is taken parallel to a $C_{2}$ axis. An example of this type is shown in Fig. 3: the crystal is a columnar hexagonal mesophase oriented with the columns perpendicular to the glass plates. For such a system the surface energy is

$$
\begin{aligned}
\gamma(\alpha, \beta)= & \left(1+\epsilon_{2}\right) \sin ^{2} \alpha+\left(1-\epsilon_{2}\right)\left(1+\epsilon_{6} \cos 6 \beta\right) \cos ^{2} \alpha \\
= & {\left[\left(1+\epsilon_{2}\right) \sin ^{2} \alpha+\left(1-\epsilon_{2}\right) \cos ^{2} \alpha\right] } \\
& +\epsilon_{6}\left[\left(1-\epsilon_{2}\right) \cos ^{2} \alpha\right] \cos 6 \beta
\end{aligned}
$$

and the correction to the shape anisotropy is given by

$$
\lim _{\substack{\rho_{0} \rightarrow \infty \\ \epsilon_{6} \rightarrow 0}} \frac{\epsilon_{6}^{\prime}-\epsilon_{6}}{\epsilon_{6}}=\epsilon_{2} \frac{4 \alpha_{0}+3 \epsilon_{2} \sin 2 \alpha_{0}-\sin 4 \alpha_{0}-\epsilon_{2} \sin 6 \alpha_{0}}{4\left(2-3 \epsilon_{2}^{2}\right) \alpha_{0}+\left(4+8 \epsilon_{2}-15 \epsilon_{2}^{2}\right) \sin 2 \alpha_{0}+\left(4-3 \epsilon_{2}\right) \epsilon_{2} \sin 4 \alpha_{0}+\epsilon_{2}^{2} \sin 6 \alpha_{0}} .
$$

The shape anisotropy thus depends on $\epsilon_{2}$ and on the contact angle $\alpha_{0}$. It is plotted as a function of $\alpha_{0}$ and $\epsilon_{2}$ in Fig. 4. Notice that to first order in $\epsilon_{6}$, the shape anisotropy equals the surface-free-energy anisotropy $\forall \alpha_{0}$ for $\epsilon_{2}=0$. When $\epsilon_{2}$ $\neq 0$, the result does not depend on the sign of the contact angle $\alpha_{0}$, and the relative variation $\left(\epsilon_{6}^{\prime}-\epsilon_{6}\right) / \epsilon_{6}$ is maximal when one of the two phases completely wets the other (i.e., when $\alpha_{0}= \pm \pi / 2$ ). This variation equals $20 \%$ for the maximal value of $\epsilon_{2}$ (i.e., $\epsilon_{2}= \pm \frac{1}{3}$ ). We recall that for $\left|\epsilon_{2}\right|>\frac{1}{3}$ there exists an angular point on the meniscus profile.

In conclusion, we have determined the shift between the surface-free-energy anisotropy and the shape anisotropy in the limit of large radius $\rho_{0}$ and vanishing anisotropy $\epsilon_{n}$. In Sec. IV, we compute shape anisotropy $\epsilon_{n}^{\prime}$ as function of the radius $\rho_{0}$ in the limit $\epsilon_{n} \ll 1$.

\section{ASYMPTOTIC BEHAVIOR FOR VANISHINGLY SMALL ANISOTROPY AND FINITE RADIUS}

Let us now consider the case of a crystal of finite radius $\rho_{0}$ and of vanishingly small anisotropy $\epsilon_{n} \rightarrow 0$. As we expect the shape anisotropy to be small, we write

$$
\rho(\theta, z)=r_{0}(z)\left[1+\epsilon_{n} r_{1}(z) \cos n \theta\right] \text {. }
$$

By definition, we have $\epsilon_{n}^{\prime}=\epsilon_{n} r_{1}(0)$ and $\rho_{0}=r_{0}(0)$. To calculate $\epsilon_{n}^{\prime}$, we replace $\rho(\theta, z)$ in Eqs. (8) and (9) and we expand them in $\epsilon_{n}$ and $\epsilon_{n}^{\prime}$ to first order. This procedure gives a second-order differential equation for $r_{0}(z)$ and boundary conditions that are independent of $r_{1}(z)$. This zero-order solution describes the isotropic meniscus. It, and the Lagrange parameter $\lambda$, corresponding to a given value of $\rho_{0}$, are obtained by using a classical shooting method [26]. Knowing $r_{0}(z)$, we then solve the second differential equation in $r_{1}(z)$ with the corresponding boundary conditions. The same shooting method can be used, the unknown parameter being now $r_{1}(0)$ (i.e., $\epsilon_{n}^{\prime}$ ).

For a uniaxial crystal in planar orientation, the shape anisotropy equals that of the surface free energy for all $\alpha_{0}$. The expansion (which we shall not give here) shows that $r_{1}(z)$ $=1$ is the solution for $\epsilon_{n} \rightarrow 0$. For a cubic or a hexagonal 


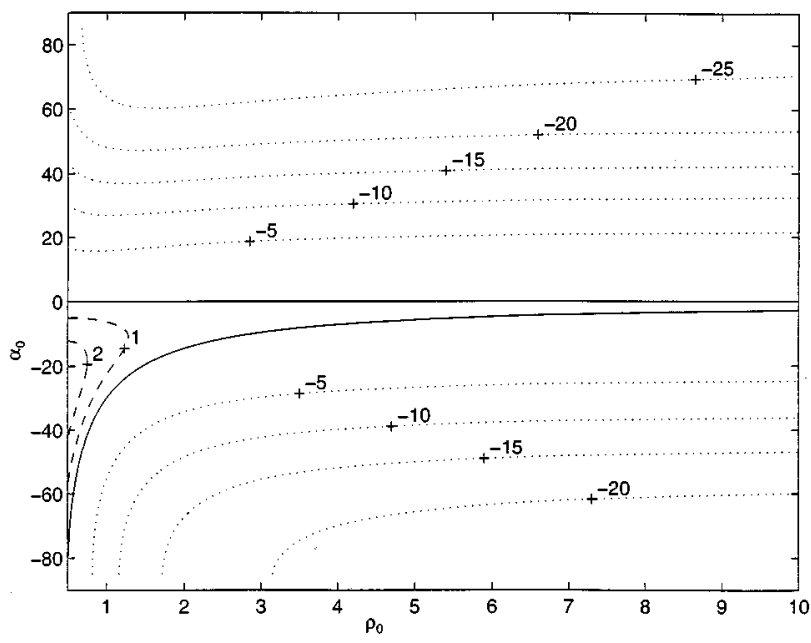

FIG. 5. Relative shape anisotropy $\left(\epsilon_{4}^{\prime}-\epsilon_{4}\right) / \epsilon_{4}(\%)$ of a cubic crystal calculated in the limit $\epsilon_{4} \rightarrow 0$. Its value, indicated on each level line, is equal to zero on the two solid lines.

crystal (Figs. 5 and 6), the numerical results show that the relative difference between the shape anisotropy and the surface energy anisotropy $\left(\epsilon_{n}^{\prime}-\epsilon_{n}\right) / \epsilon_{n}$ is usually small (of the order of a few \%). The asymptotic value corresponding to
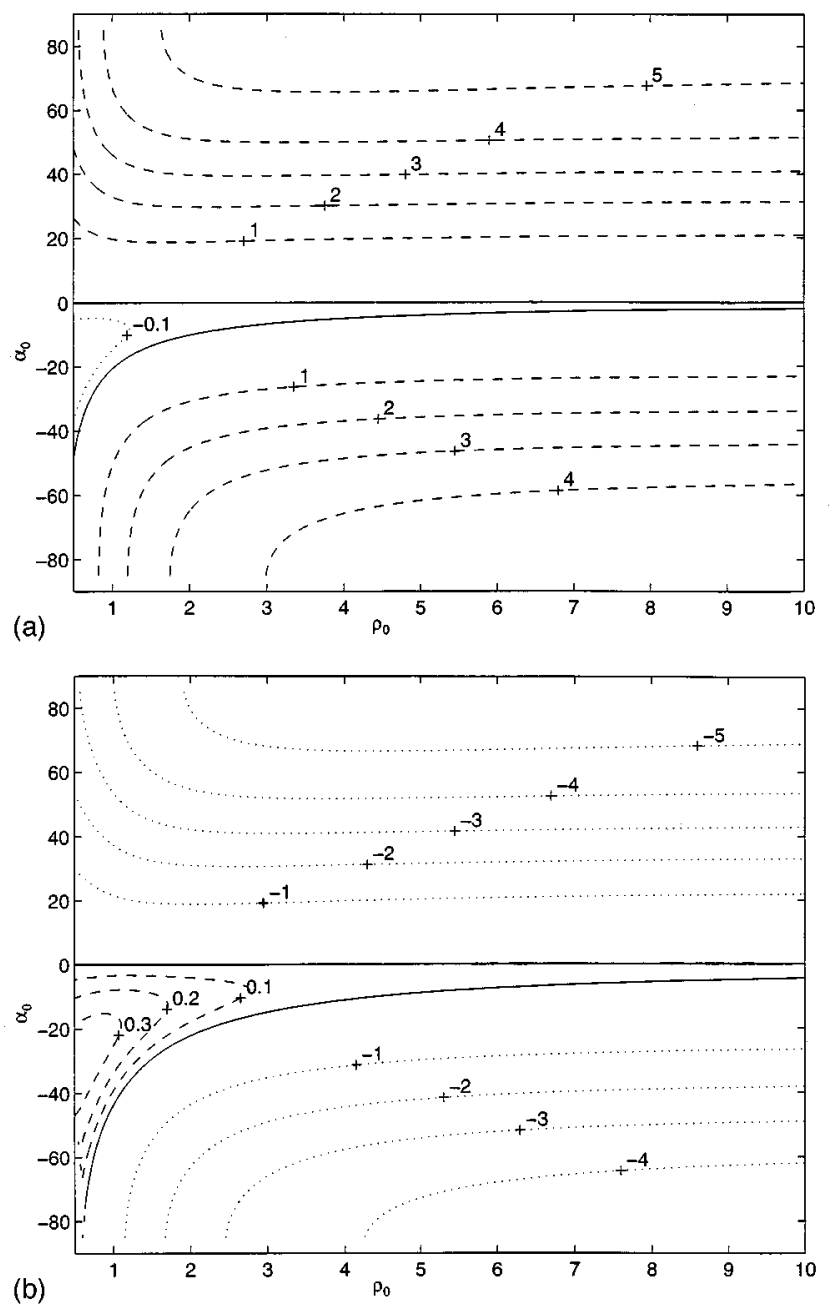

FIG. 6. Relative shape anisotropy $\left(\epsilon_{6}^{\prime}-\epsilon_{6}\right) / \epsilon_{6}(\%)$ of a hexagonal crystal in the limit $\epsilon_{6} \rightarrow 0$. (a) $\epsilon_{2}=0.1$. (b) $\epsilon_{2}=-0.1$.

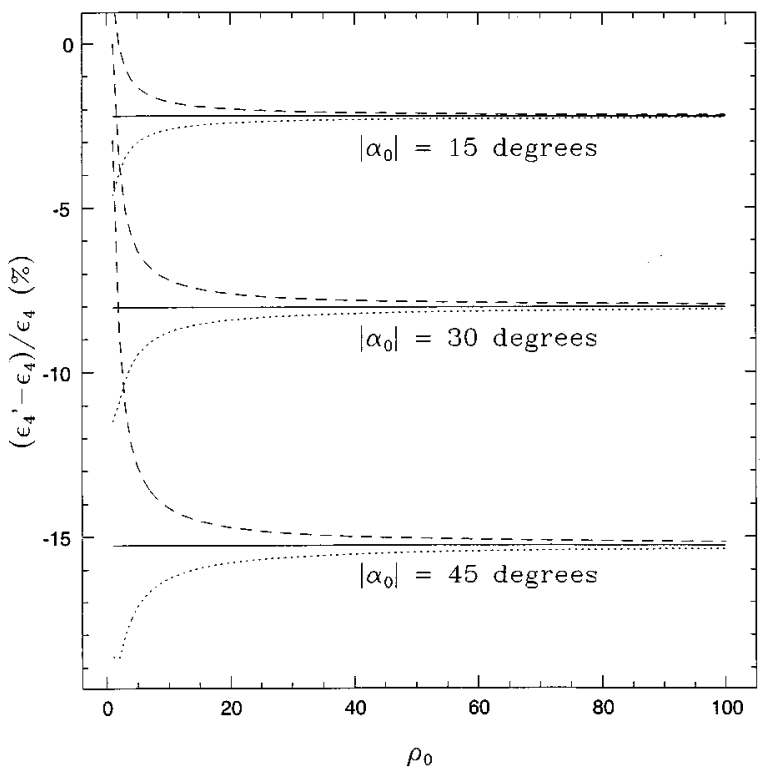

FIG. 7. Behavior of $\left(\epsilon_{4}^{\prime}-\epsilon_{4}\right) / \epsilon_{4}(\%)$ as a function of the radius $\rho_{0}$ when $\epsilon_{4} \rightarrow 0$. (The solid lines give the limits $\rho_{0} \rightarrow \infty$ [Eq. (34)]. The short-dashed lines correspond to $\alpha_{0}>0$ and the long-dashed lines, to $\alpha_{0}<0$.) Note that $\left(\epsilon_{4}^{\prime}-\epsilon_{4}\right) / \epsilon_{4}$ tends to its asymptotic value like $1 / \rho_{0}$.

$\rho_{0} \rightarrow \infty$ is reached (to $\pm 1 \%$ ) when the radius $\rho_{0}$ is larger than about 20 times the sample thickness (Fig. 7). For small radii $\rho_{0}$, the coupling between the in-plane curvature and the curvature in the thickness is asymmetric in $\alpha_{0}$. As a consequence, the correction depends on the sign of $\alpha_{0}$. The results are given only for $\rho_{0}>0.5$ because the meniscus can be linearly unstable when the radius $\rho_{0}$ is smaller than typically the half distance between the limiting walls [27].

\section{CRYSTAL OF FINITE ANISOTROPY $\epsilon_{n}$ AND OF FINITE RADIUS $\rho_{0}$}

In this last section, we extend the numerical method of Sec. IV to the case of a uniaxial crystal of planar orientation [see Eq. (31)]. Equations (8) and (9) are expanded to third order in $\epsilon$ and yield four second-order differential equations for the $r_{i}(z)$ :

$$
\rho(\theta, z)=r_{0}(z)\left(1+\sum_{k=1}^{\infty} \epsilon_{n}^{k} r_{k}(z) \cos k n \theta\right) .
$$

A shooting method is used to determine the midplane values $r_{i}(0)$, which satisfy the boundary conditions on the limiting walls. The numerical results show that the error made in solving this problem to $O(m)$, scales like $\epsilon_{2}^{m}$.

We have previously shown that the shape anisotropy tends to the surface-free-energy anisotropy when $\epsilon_{2} \rightarrow 0$. The numerical results (Fig. 8) now show that $\left(\epsilon_{2}^{\prime}-\epsilon_{2}\right) / \epsilon_{2}$ remains small (a few \%) even for large $\epsilon_{2}$. They also confirm that, as expected, the difference scales like $\epsilon_{2}$.

\section{CONCLUDING REMARKS}

The equilibrium shape of an anisotropic crystal confined between two planar parallel walls depends on its wetting 

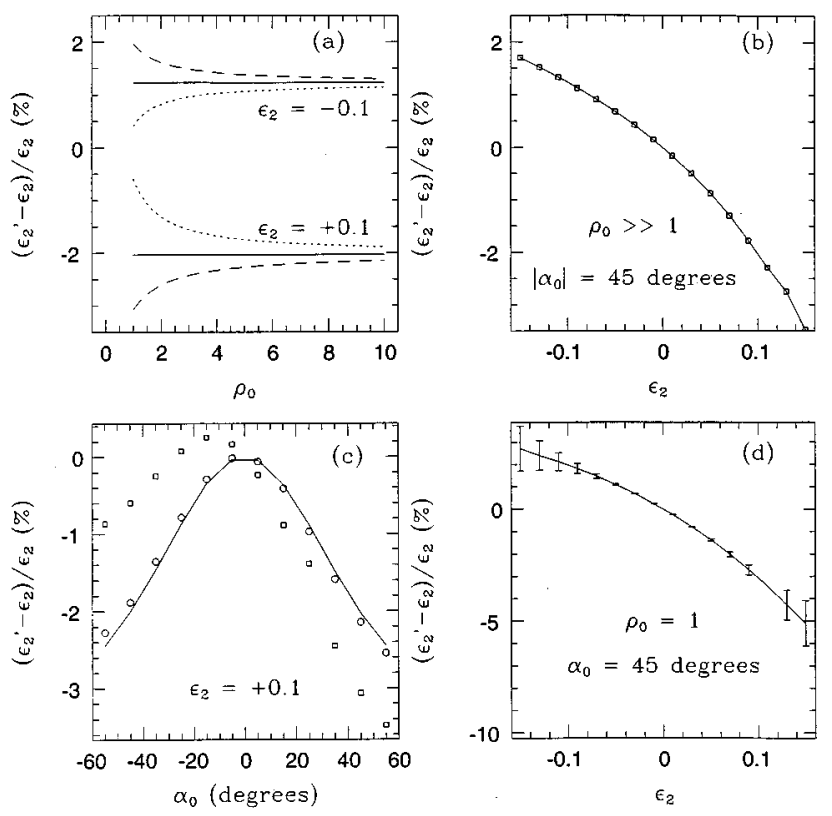

FIG. 8. Numerical results for an uniaxial crystal in planar orientation. (a) Relative shape anisotropy $\left(\epsilon_{2}^{\prime}-\epsilon_{2}\right) / \epsilon_{2}$ as a function of the radius $\rho_{0}$ for $\alpha_{0}=+45^{\circ}$ (short-dashed line), $\alpha_{0}=-45^{\circ}$ (longdashed line), and $\epsilon_{2}= \pm 0.1$. The solid lines give the asymptotic values at large radius $\rho_{0} \rightarrow \infty$. (b) Asymptotic value of $\left(\epsilon_{2}^{\prime}\right.$ $\left.-\epsilon_{2}\right) / \epsilon_{2}$ as a function of $\epsilon_{2}$ for $\left|\alpha_{0}\right|=45^{\circ}$ and $\rho_{0} \rightarrow \infty$. (c) Relative shape anisotropy $\left(\epsilon_{2}^{\prime}-\epsilon_{2}\right) / \epsilon_{2}$ as a function of $\alpha_{0}$ for $\epsilon_{2}$ for $\epsilon_{2}$ $=0.1$ and $\rho_{0}=1$ (squares), $\rho_{0}=10$ (circles), and $\rho_{0} \rightarrow \infty$ (solid line). (d) $\left(\epsilon_{2}^{\prime}-\epsilon_{2}\right) / \epsilon_{2}$ as a function of $\epsilon_{2}$ for $\left|\alpha_{0}\right|=45^{\circ}$ and $\rho_{0}=1$.

conditions and on the shape of the meniscus that forms in the sample thickness. The anisotropy of the vertical part of the meniscus, which is observable in the microscope, does not equal the surface-free-energy anisotropy in the plane of the limiting walls and depends on the size of the germ.

When the radius of the germ is much larger than the sample thickness and when the anisotropy vanishes, the shape anisotropy tends to an asymptotic value that depends on the contact angle $\alpha_{0}$ but not on its sign. In addition, this asymptotic value is reached within $1 \%$ when the radius $\rho_{0}$ is larger than about 20 times the sample thickness. We emphasize that the shape of the meniscus in this limit is again self-similar. On the other hand, the asymptotic value of $\epsilon_{n}^{\prime}$ is different from the value of the anisotropy of the energy per unit length of the meniscus. Indeed, one could be tempted to calculate the energy $\Gamma(\beta)$ per unit length (integrated over the thickness) of a straight meniscus and then to use the corresponding 2D Wulff construction. This procedure leads to a wrong result because curving the meniscus in the midplane while keeping its shape unchanged in the thickness does not maintain a constant pressure difference $\Delta P=-\lambda$ across the whole interface.

Experimentally, we observed that a germ's anisotropy usually does not depend on its size and on the sign of the contact angle (by taking a germ of solid in the liquid and a droplet of liquid in the solid). We wrongly concluded that the wetting condition had no effect on our measurement of surface-free-energy anisotropy [28]. However, in the discotic liquid crystal shown in Fig. 3, the radii of the germs (typically $20-50 \mu \mathrm{m}$ ) are larger than the sample thickness (typically $2-10 \mu \mathrm{m}$ for Ref. [28]) and the asymptotic value is reached within a few percent. The experimental errors do not allow us to determine the dependence of the shape anisotropy on the radius and on the contact angle. The present work shows that our systematic error on the value of $\epsilon_{6}$ for the hexagonal phase (Fig. 7) is of about $5 \%\left(\epsilon_{2} \sim 0.1\right)$ and thus negligible: This small systematic error cannot explain the discrepancy between the experimental value of the stability constant of the dendrites $\sigma_{2 \mathrm{D}}^{*}=0.041$ and its "theoretical" value $\sigma_{2 \mathrm{D}}^{*}=0.033$ [29]. Useful analytical expressions of the asymptotic value of the shape anisotropy are provided in the present article.

For finite anisotropy, the discrepancy between the shape anisotropy and the surface energy anisotropy is about $10 \%$. This discrepancy is however not large enough to explain the often encountered disagreement between the experimental and theoretical values of the stability constant of stationary dendrites.

\section{ACKNOWLEDGMENT}

This work has been supported by DRET Contract No. 95.1117.
[1] G. Wulff, Z. Kristallogr. 34, 449 (1901).

[2] L. D. Landau and E. M. Lifshitz, Statistical Physics (Pergamon, Oxford, 1980).

[3] R. Kern, Morphology of Crystal, A, edited by I. Sunagawa (Reidel, Dordrecht, 1987).

[4] Ph. Nozières, in Solids far from Equilibrium, edited by C. Godrèche (Cambridge University Press, Cambridge, 1992).

[5] P. W. Voorhees, S. R. Coriell, G. B. McFadden, and R. F. Sekerka, J. Cryst. Growth 67, 425 (1984).

[6] G. E. Volovik and O. D. Lavrentovich, Zh. Eksp. Teor. Fiz. 85, 1997 (1983) [Sov. Phys. JETP 58, 1159 (1983)].

[7] W. Huang and G. F. Tuthill, Phys. Rev. E 49, 570 (1994).

[8] Y. Bouligand, J. Phys. (France) 33, 525 (1972).
[9] G. Friedel and F. Grandjean, Bull. Soc. Fr. Mineral Cristallogr. 33, 409 (1910).

[10] J. B. Fournier and G. Durand, J. Phys. (France) II 1, 845 (1991).

[11] M. V. Kurik and O. D. Lavrentovich, Zh. Eksp. Teor. Fiz. 85, 511 (1983) [Sov. Phys. JETP 58, 299 (1983)].

[12] Y. Bouligand, J. Phys. (France) 41, 1297 (1980); 41, 1307 (1980).

[13] M. Kléman, J. Phys. (France) 41, 737 (1980).

[14] P. Oswald and M. Kléman J. Phys. (France) 42, 1461 (1981).

[15] J. B. Fournier, Phys. Rev. Lett. 75, 854 (1995).

[16] J. R. Rudnick and R. Bruinsma, Phys. Rev. Lett. 74, 2491 (1995). 
[17] P. Galatola and J. B. Fournier, Phys. Rev. Lett 75, 3297 (1995).

[18] The 2D parametric equation of the interface, deduced from the Wulff construction [2-4], is given by Eqs. (21) and (22). If there is no angular point on the interface, the radius $r$ in a plane of symmetry (where $\gamma^{\prime}=0$ ) reduces to $r=-\gamma / \lambda$. Thus, the maximal and minimal values of the radius $\left(r_{\max }\right.$ and $r_{\min }$, reached in planes of symmetry of the crystal) and the maximal and minimal values of the surface free energy $\left(\gamma_{\max }\right.$ and $\left.\gamma_{\text {min }}\right)$ satisfy $\epsilon^{\prime}=\left(r_{\max }-r_{\min }\right) /\left(r_{\max }+r_{\min }\right)=\left(\gamma_{\max }-\gamma_{\min }\right) /\left(\gamma_{\max }\right.$ $\left.+\gamma_{\text {min }}\right)=\epsilon$. The surface-shape anisotropy $\epsilon$ equals the surfacefree-energy anisotropy $\epsilon^{\prime}$.

[19] P. Pelcé, Dynamics of Curved Fronts (Academic, New York, 1988).

[20] D. A. Kessler, J. Koplik, and H. Levine, Adv. Phys. 37, 255 (1988).

[21] E. A. Brener and V. I. Mel'nikov, Adv. Phys. 40, 53 (1991).

[22] H. Müller-Krumbhaar and W. Kurz, in Phase Transformations in Materials, edited by P. Haasen (VCH-Verlag, Weinheim, 1991).

[23] J. P. Gollub, in Growth and Form, edited by M. Ben Amar et al. (Plenum, New York, 1991).

[24] M. Muschol. D. Liu, and H. Z. Cummins, Phys. Rev. A 46, 1038 (1992).

[25] J. De Coninck and F. Dunlop, J. Stat. Phys. 47, 827 (1987).

[26] W. H. Press, B. P. Flannery, S. A. Teukolsky, and W. T. Vetterling, Numerical Recipes (Cambridge University Press, Cambridge, 1986).

[27] D. Strube, Microgravity Sci. Technol. 5, 56 (1992).

[28] J. C. Géminard, Thèse, Université Claude Bernard Lyon I France (1993).

[29] J. C. Géminard and P. Oswald, J. Phys. (France) II 4, 959 (1994).

[30] N. H. Hartshorne and A. Stuart, Crystals and the Polarising Microscope (Arnold, London, 1970). 